\title{
Clinical and Biological Characteristics of Breast Cancer in Women with Mammary Paget's Disease: A Retrospective Study of 36 cases
}

\author{
Mahmoud Al-Balas ${ }^{1 *}$, Margherita Serra ${ }^{2}$, Hamzeh Al-Balas ${ }^{1}$, Donatella Santini ${ }^{3}$ and Mario Taffurelli ${ }^{2}$
}

\author{
*Corresponding author: \\ Mahmoud Al-Balas, MBBS \\ Department of General and Special \\ Surgery, Faculty of Medicine \\ The Hashemite University \\ P.0. Box 330127, Zarqa 13133 \\ Jordan \\ E-mail: Mahmoud_albalas@hu.edu.jo
}

\author{
'Department of General and Special Surgery, Faculty of Medicine, Hashemite University, \\ Zarqa, Jordan \\ ${ }^{2}$ Department of the Health of Woman, Child and Urological Diseases, \\ S. Orsola-Malpighi Hospital, University of Bologna, Bologna, Italy \\ ${ }^{3}$ Pathology Unit, S.Orsola-Malpighi Hospital, Bologna, Italy
}

\section{ABSTRACT}

Objective: Identification of clinical and biological characteristics of breast cancer in women with mammary Paget's disease (MPD).

Methods: We performed a retrospective analysis for 36 women with a primary diagnosis of mammary Paget's disease, patients were treated in Sant' Orsola-Malpighi Breast Unit in Italy, between 2000 and 2016. Patients demographics, clinical data, radiologic and pathologic reports were extracted from electronic health records.

Result: 36 cases of patients with a diagnosis of MPD were involved. The mean age was 64.28 years (Range, $36-86$ years). Only 14 patients $(38.9 \%)$ were found to have an underlying breast mass. Invasive breast carcinoma was diagnosed in 23 patients (63.9\%), 7 patients (19.4\%) had ductal carcinoma in situ. All patients with associated breast mass were diagnosed with invasive breast carcinoma and they had a higher rate of axillary lymph node metastasis. Most invasive breast carcinomas were HER2 positive (65.2\%), estrogen and progesterone receptors negative (56.5\% and 69.6\%).

Conclusion: Mammary Paget's disease is a rare type of cancer that can be localized to nipple-areola complex or it can be associated with underlying breast carcinoma. Breast MRI has a higher sensitivity for identifying underlying breast pathology. Once MPD is associated with an underlying mass, the likelihood of carcinoma is higher so a biopsy is recommended. The expression of ER/PR is lower in breast carcinomas with MPD, while they have higher HER2/neu expression. Unless multifocality or multicentricity is excluded, mastectomy is the recommended surgical treatment. Sentinel lymph node biopsy should be performed to evaluate the axilla when the invasive disease is identified or a mastectomy is planned.

Key words: invasive breast carcinoma, mammary Paget's disease (MPD), Nipple Dermatitis

\section{INTRODUCTION}

Accepted: 04.12 .2020

$\overline{\text { Copyright } \odot \text { Celsius Publishing House }}$ www.sgo-iasgo.com
Mammary Paget's disease (MPD) of the breast is a rare type of cancer of the nipple-areola complex that was first described in 1874 by Sir James Paget and accounts for approximately $1-4.3 \%$ of all breast cancers $(1,2)$, it is 
often associated with underlying invasive or in situ carcinoma. It is defined clinically as a chronic, eczematous rash of the nipple and adjacent areolar tissue that is more commonly encountered in older postmenopausal women (mean age 57 years) (3). Two patterns of MPD are identified based on physical examination (4); with or without underlying breast mass, while it is classified pathologically into three patterns: MPD with underlying invasive carcinoma, MPD with underlying ductal carcinoma in situ (DCIS) and MPD without underlying pre-cancerous or cancerous conditions of the breast (5).

The prevalence of MPD associated carcinoma ranges from $67 \%$ to $100 \%$, the presence of underlying breast mass or parenchymal pathology makes the probability of diagnosing invasive carcinoma higher in comparison to patients without breast mass $(6,7,8)$.

As Paget's disease of the breast usually presents as a thickened, eczematous, erythematous or crusted lesion of the nipple and adjacent areola, it can be easily mistaken for eczema or other inflammatory nipple conditions, so treatment with topical steroids is falsely practiced by physicians in these cases regardless transient improvement that can be noticed $(9,10)$. Manifestations of pain, itching, nipple discharge or nipple deformity are also frequent.

\section{MATERIALS AND METHODS}

We reviewed the medical reports of all patients with clinical and histological diagnosis of Mammary Paget's Disease who were treated in Sant' Orsola-Malpighi Breast Unit, between 2000 and 2016. The clinical presentation, menstrual status, personal and family history of breast cancer, pregnancy history, and smoking status were all extracted from the case notes.

Mammographic and ultrasound reports were reviewed to identify cases of MPD with an associated underlying mass. Operative reports as well as final pathological reports were evaluated for the type of procedure, lymph node status, and clinicopathological classification of associated carcinoma.

Our study aims to evaluate differences between MPD cases in the presence or absence of an underlying breast parenchymal pathology.

\section{RESULTS}

In the period between 2000 and 2016, a total of 3571 cases of invasive breast carcinoma and 484 cases with carcinoma in situ were diagnosed in our hospital. Only thirty-six cases of women with MPD were collected from our hospital records. The mean age was 64.28 years (Range, 36-86 years). Breast mass was identified in 14 cases (38.9\%) while 22 cases (61.1\%) didn't have an underlying mass. Although it was not statistically significant, the mean age for cases with an associated mass was found to be less than cases without an associated mass (59.57 vs. 67.27 years). The Mean age of menarche was 12.53 years. $30.3 \%$ of women were pre-menopausal and the mean age of menopause was 49.7 years (Range, $32-55$ years). Smoking was prevalent in $27.7 \%$ of all Paget's cases. A personal history of breast cancer was found in 3 patients of MPD with a mass group (21.4\%), and in 6 patients of Paget's only disease (27.3\%). Only one patient of Paget's with mass had a family history of breast cancer while five patients $(22.7 \%)$ had this risk factor in Paget's only disease group. Regardless of all preceding figures, there were no statistically significant differences between both groups. $22.2 \%$ of women with MPD were nulliparous, and $16.6 \%$ had their first pregnancy at the age of 30 or older. Table 1 illustrates general features for patients with MPD.

Clinically, 11 patients had clinically palpable breast mass in association with MPD while in 3 patients, breast parenchymal mass or distortion was identified using breast mammogram and ultrasound. Patients were labeled as Paget's only disease when we failed to demonstrate masses or parenchymal distortion either clinically or radiologically. Nipple eczema or ulceration was the most frequent clinical sign in clinically diagnosed Paget's disease; 32 women experienced significant nipple changes (88.9\%) while 4 women (11.1\%) have only very little changes that were not recognized by their practitioners. Nipple discharge was noticed in $44.4 \%$ of cases (16/36). Seven patients in Paget's with the mass group had spontaneous nipple

Table 1 - General features for patients with MPD

\begin{tabular}{lc}
\hline Number of Cases & 36 \\
\hline $\begin{array}{l}\text { Site of pathology } \\
\text { Right } \\
\text { Left }\end{array}$ & 24 \\
\hdashline Menarche age (mean) & 12 \\
\hline Menopause age (mean) & 12.53 \\
\hline Smoking & 49.7 \\
\hline Personal history of Breast Cancer & $10 / 36(27.7 \%)$ \\
\hline Family History of Breast Cancer & $9 / 36(25 \%)$ \\
\hline Nulliparity & $6 / 36(16.6 \%)$ \\
\hline 1st Pregnancy $<30$ years & $8 / 36(22.2 \%)$ \\
\hline 1st Pregnancy $\geq 30$ years & $21 / 36(58.3 \%)$ \\
\hline
\end{tabular}


discharge (50\%) while 9 women $(40.9 \%)$ experienced it in Paget's only group. Palpable axillary lymphadenopathy was found only in two patients of Paget's with mass (15.4\%) (table 2).

Mammographic findings associated with Paget's disease ranged from a normal mammogram (55.6\%), malignant microcalcifications only (13.9\%), and a mass (or parenchymal distortion) with or without microcalcifications (30.5\%). Table 3 compares the mammographic findings in both groups of PD.

\section{Surgical Management and Clinicopathological Features}

As a preoperative confirmation of diagnosis, a nipple biopsy was performed for 29 patients, and it proved the diagnosis of MPD. The remaining 7 patients were diagnosed clinically and histopathologic diagnosis were confirmed retrospectively. Out of 16 women with pathologic nipple discharge, cytological examination of this discharge was ordered for 14 patients, the presence of malignant cells have been proved in 11 of them.

Mastectomy was the procedure of choice for 32 cases (89\%) and central quadrantectomy in 4 cases only (11\%). In the mastectomy group, 22 patients had mastectomy alone, 2 patients had a mastectomy with expander while the remaining 8 patients performed skin-sparing mastectomy with an expander/implant reconstruction.

A total of 23 patients had a final diagnosis of invasive ductal carcinoma (IDC) in association with MPD, the majority of them were labeled based on the AJCC 8th edition TNM staging system as T1 tumors ( $n=20,87 \%)$. All patients with a mass $(n=14)$ were found to have an underlying IDC, three of them (21.4\%) had ductal carcinoma in situ (DCIS) in association with their invasive component. In comparison, only 9 patients (41\%) of MPD patients without a mass had an invasive carcinoma ( $P=0.0012) .7$ patients of this group $(31.8 \%)$ had only DCIS and 6 patients (27.2\%) had nipple Paget's that was limited only to the dermis without an underlying carcinoma table 4.

Sentinel lymph node biopsy was utilized as a procedure for axillary staging in 26 patients (72.2\%), and 6 patients had ALND without SLNB. The axillary staging was omitted in 3 patients due to their previous history of breast cancer and ALND and in one patient with a history of DCIS because of its advanced age and poor medical condition. Sentinel lymph node macrometastasis was found in 3 patients (11.5\%), micrometastasis in only one patient (3.84\%), and all of
Table 2 - Clinical presentation of mammary Paget's disease (MPD)

\begin{tabular}{lrr}
\hline Clinical Presentation & $\begin{array}{r}\text { MPD with a mass } \\
(\mathbf{N}=\mathbf{1 4})\end{array}$ & $\begin{array}{r}\text { MPD without a mass } \\
(\mathbf{N}=\mathbf{2 2})\end{array}$ \\
\hline Palpable breast mass & 11 & 0 \\
\hline $\begin{array}{l}\text { Nipple changes } \\
\text { (eczema / ulceration) }\end{array}$ & $12(85.7 \%)$ & $20(90.9 \%)$ \\
\hline $\begin{array}{l}\text { Nipple Discharge } \\
\text { (Bloody) }\end{array}$ & $7(50 \%)$ & $9(40.9 \%)$ \\
\hline $\begin{array}{l}\text { Axillary } \\
\text { Lymphadenopathy }\end{array}$ & $2(14.3 \%)$ & 0 \\
\hline
\end{tabular}

Table 3 - Mammographic features of mammary Paget's disease

\begin{tabular}{lcr}
\hline $\begin{array}{l}\text { Mammographic } \\
\text { Features }\end{array}$ & $\begin{array}{r}\text { MPD with a mass } \\
\mathbf{( N = 1 4 )}\end{array}$ & $\begin{array}{r}\text { MPD without a mass } \\
\mathbf{( N = 2 2 )}\end{array}$ \\
\hline Normal & $1(7.1 \%)$ & $19 / 22(86.4 \%)$ \\
\hline Microcalcifications & $2(14.3 \%)$ & $3 / 22(13.6 \%)$ \\
\hline Mass/Parenchymal distortion & $9(64.3 \%)$ & 0 \\
\hline Mass with microcalcification & $2(14.3 \%)$ & 0 \\
\hline
\end{tabular}

(MPD): Mammographic findings

them were operated with an ALND subsequently. 50\% of patients who performed axillary node dissection as the primary procedure for axillary staging were found positive for metastasis. Axillary lymph node metastasis was more common in patients with a mass (38.5\%) than those without a mass (5.5\%), $P=0.029$.

Among patients with a diagnosis of IDC $(n=23)$, Estrogen receptors (ER) were positive in 10 patients (43.5\%), 7 patients (30.4\%) were PR positive and 15 patients (65.2\%) were HER2 positive. Table 5 shows the biologic classification of MPD associated IDC in our patient group.

\section{DISCUSSION}

MPD is often mistaken for a benign dermatologic condition such as nipple dermatitis. Once the diagnosis is suspected, a skin biopsy of the eczematous lesion should be obtained. Nipple biopsy could be in a form of wedge biopsy, superficial "shave" biopsy of the epidermis, or punch biopsy (3). No one of these methods is always successful, so a second biopsy or

Table 4 - Histopathologic findings in MPD

\begin{tabular}{lcc}
\hline & $\begin{array}{c}\text { MPD with a mass } \\
(\mathbf{N}=\mathbf{1 4})\end{array}$ & $\begin{array}{c}\text { MPD without a mass } \\
(\mathbf{N}=22)\end{array}$ \\
\hline IDC & $14^{*}$ & 9 \\
\hdashline DCIS only & 0 & 7 \\
\hline Nipple Paget's only & 0 & 6 \\
\hline
\end{tabular}

${ }^{\star}$ Additional DCIS have been demonstrated in 3 cases 
Table 5 - Biologic classification of MPD associated IDC $(n=23)$

\begin{tabular}{lc}
$\begin{array}{l}\text { Biologic } \\
\text { Subtype * }\end{array}$ & $\begin{array}{r}\text { Number of cases } \\
\text { (\%) }\end{array}$ \\
\hline $\begin{array}{l}\text { Luminal A } \\
\text { (ER and/or PR positive, Her2 negative, Ki-67 <14\%) }\end{array}$ & $4(17.4 \%)$ \\
\hline $\begin{array}{l}\text { Luminal B (HER-2 negative) } \\
\text { (ER and/or PR positive, Her2 negative, Ki-67 >=14\%) }\end{array}$ & $3(13 \%)$ \\
\hline Luminal B (HER-2 positive) & $4(17.4 \%)$ \\
(ER and/or PR positive, Her2 overexpresssed or amplified, \\
any Ki-67) \\
$\begin{array}{l}\text { HER-2 overexpression } \\
\text { (ER and PR absent, HER2 overexpressed or amplified) }\end{array}$ \\
\hline $\begin{array}{l}\text { Triple negative } \\
\text { (ER and PR absent, HER2 negative) }\end{array}$ \\
\hline ER: estrogen receptor; PR: progesterone receptor, \\
HER2: human epidermal growth factor receptor 2
\end{tabular}

nipple excision may be performed to reach a diagnosis (10). A wedge biopsy is the most useful method because it can show sufficient epidermis as well as lactiferous ducts (3). The cytological examination is not sufficient to confirm the MPD diagnosis.

Once the diagnosis of MPD is confirmed, further imaging studies are needed to exclude underlying breast parenchymal pathology as the presence of a mass is linked to a higher probability of underlying breast carcinoma. The reported multifocality and multicentricity of breast carcinoma prevalence in MPD are $41 \%$ and $34 \%$, respectively (11). Ultrasound and mammography sensitivity for detecting IDC multifocality or multicentricity in MPD is $79 \%$ and $74 \%$ respectively while it is lower for DCIS (39\% and $19 \%$ respectively). On the other hand, MRI was shown to be $100 \%$ sensitive for IDC and $44 \%$ for DCIS, so it can help better in identifying patients with localized diseases $(12,13,14)$. If a lesion is identified, biopsy should be taken for further assessment.

The surgical treatment of MPD is controversial. Because of its low incidence, no randomized studies are evaluating the optimal surgical management (15). Mastectomy with or without ALND was the standard surgical treatment of MPD (3). The reason behind this approach is the high association either with IDC or DCIS (14). Kothari and colleagues showed that 60\% of patients with MPD having underlying invasive carcinoma, more than $40 \%$ of those had multicentric and/or multifocal disease and more than $60 \%$ had axillary lymph node involvement (11). In a metaanalysis including 7 studies comparing the local recurrence of MPD after mastectomy and breast conservative surgery (BCS), the local recurrence rate in the mastectomy group was $5.6 \%$ while it was $13.2 \%$ in the BCS group. The pooled OR for local recurrence was
0.38 (95\% Cl 0.21-0.69). It was shown that relapse cases tend to develop invasive carcinoma with poor prognosis after previous BCS (16). According to surveillance, epidemiology, and end results (SEER) data for patients with MPD during 2000 - 2011 (17), 2631 patients were identified. Of these patients, $7 \%$ had MPD of the nipple only, $36.2 \%$ had MPD with DCIS (MPD-DCIS) and $56.7 \%$ had MPD with invasive ductal carcinoma (MPD-IDC). The overall rates of mastectomy for these groups were $47,69,88.9 \%$ respectively.

Axillary lymph node metastasis was observed more in MPD patients with underlying breast mass (18). According to Wong SM et al. in their cohort study for the SEER database for MPD-IDC patients (17), MPD-IDC had higher odds of axillary lymph node metastasis in comparison to patients with IDC alone (OR 1.83; $\mathrm{P}<0.001)$. Retrospective studies regarding the use of sentinel lymph node biopsy in cases of MPD showed that nearly $11 \%$ of cases were positive for metastasis even in the absence of radiologic features of breast cancer, so SLNB should be performed to evaluate the axilla when the invasive disease is identified or a mastectomy is planned $(19,20)$.

In comparison to other invasive breast carcinomas, MPD-IDC showed larger tumor size, more multifocal disease, lower ER/PR expression, and higher HER2 overexpression $(21,22)$. In our study, ER, PR and HER2 were positive in $43.5 \%, 30.4 \%$, and $65.2 \%$ of MPD associated carcinomas respectively. In comparison, both ER/PR were positive in more than $75 \%$ of patients and HER2 is lower than $40 \%$ in patients without MPD. Based on Arsenal et al data, MPD-IDC patients showed expression of ER and PR in $38.5 \%$ and $33.3 \%$, respectively, and HER2 overexpression in $41 \%$ (23). In another study, overexpression of the HER2/neu (c-erb-B2) oncogene was detectable in $83 \%$ of cases (11).

Patients with MPD with associated underlying breast carcinoma have been shown to have poor survival. The 5 and 10-year survival rates have been reported to be $32-43 \%$ and $31-49 \%$, respectively (24). In one study conducted by Kothari, the 10-year overall survival for MPD with IBC was $49 \%$ while for patients with IBC alone it was $64 \%$ (11). This poor survival was attributed to the high incidence of HER2/neu positive cells, but this difference was eliminated if they were also matched for HER2 status.

MPD had been reported by several investigators as the first local recurrence event after breastconservative surgery or nipple-sparing mastectomy. In one study, seven cases of MPD were reported as a recurrence after nipple-sparing mastectomy, which accounted for $19.4 \%$ of total recurrences (25), one 
explanation for this is the possible existence of MPD before the operation.

\section{CONCLUSION}

Mammary Paget's disease is a rare disease that can be localized to nipple-areola complex or it can be associated with underlying breast carcinoma. According to the literature, breast MRI has a higher sensitivity for identifying underlying breast pathology. Once MPD is associated with an underlying mass, the likelihood of carcinoma is higher so a biopsy is recommended. The expression of ER/PR is lower in breast carcinomas with MPD, while they have higher HER2/neu expression. Unless multifocality or multicentricity is excluded, mastectomy is the recommended surgical treatment. Sentinel lymph node biopsy should be performed to evaluate the axilla when the invasive disease is identified or a mastectomy is planned.

\section{Statement conflict of Interest}

The authors declare no potential conflicts of interest with respect to the research, authorship and/or publication of this research.

\section{Funding statement}

The Authors received no financial support for the research, authorship and/or publication of this article.

\section{Conflict of interest}

No conflict of interest between authors

\section{Ethical approval}

No institutional ethical approval is required to perform descriptive retrospective studies.

\section{REFERENCES}

1. Tavassoli FA. Norwalk, Pathology of the breast; Connecticut: Appleton and Lange; 1999. p. 731-60

2. Paget J. On the disease of the mammary areola preceding cancer of the mammary gland. St Bartholomews Hosp Rep. 1874; $10: 87-9$

3. Cansu Karakas; Paget's disease of the breast. J Carcinog. 2011 10:31.

4. Sakorafas GH, Blanchard K, Sarr MG, Farley DR. Paget's disease of the breast. Cancer Treat Rev. 2001;27(1):9-18.

5. Nance FC, DeLoach DH, Welsh RA, Becker WF. Paget's disease of the breast. Ann Surg. 1970; 171(6):864-74.

6. Ashikari R, Park K, Huvos AG, Urban JA. Paget's disease of the breast. Cancer 1970;26(3):680-5.

7. Paone JF, Baker RR. Pathogenesis and treatment of Paget's disease of the breast. Cancer. 1981;48(3):825-9.

8. Zakaria S, Pantvaidya G, Ghosh K, Degnim AC. Paget's disease of the breast: accuracy of preoperative assessment. Breast Cancer Res Treat 2007;102 (2):137-142.

9. Sakorafas GH, Blanchard K, Sarr MG, Farley DR. Paget's disease of the breast. Cancer Treat Rev. 2001;27(1):9-18.

10. Rosen PP. Rosen's breast pathology. Paget's disease of the nipple; 2nd ed. Philadelphia: Lippincott-Raven; 2001. p. 565-80.

11. Kothari AS, Beechey-Newman N, Hamed H, Fentiman IS, D'Arrigo C, Hanby AM, et al. Paget disease of the nipple: a multifocal manifestation of higher-risk disease. Cancer. 2002; 95(1):1-7.

12. Siponen E, Hukkinen $K$, Heikkilä $P$, Heikki Joensuu, Marjut Leidenius Surgical treatment in Paget's disease of the breast. Am J Surg. 2010;200(2):241-6.

13. Morrogh M, Morris EA, Liberman L, Van Zee K, Cody HS 3rd, King TA. MRI identifies otherwise occult disease in select patients with Paget disease of the nipple. J Am Coll Surg. 2008; 206(2):316-21. Epub 2007 Oct 29.

14. Ana P. Lourenco, Martha B. Mainiero. Paget's disease of the breast. Rhode Island Medical Journal. 2013; p. 50-51.

15. KatarzynaTrebska-McGowan, Krista P. Terracina, and Kazuaki Takabe . Update on the surgical management of Paget's disease. Gland Surg. 2013;2(3):137-142

16. Lia YJ, Huangb XE, Zhoub XD. Local Breast Cancer Recurrence after Mastectomy and Breast-Conserving Surgery for Paget's Disease: A Meta-Analysis. Breast Care (Basel). 2014;9(6): 431-4.

17. Wong SM, Freedman RA, Stamell E, Sagara Y, Brock JE, Desantis SD, et al. Modern Trends in the Surgical Management of Paget's Disease. Ann Surg Oncol. 2015;22(10):3308-16.

18. Yim JH, Wick MR, Philpott GW, Norton JA, Doherty GM. Underlying pathology in mammary Paget's disease. Ann Surg Oncol. 1997;4(4):287-92.

19. Sukumvanich P, Bentrem DJ, Cody HS 3rd, Brogi E, Fey JV, Borgen $\mathrm{PI}$, et al. The role of sentinel lymph node biopsy in Paget's disease of the breast. Ann SurgOncol. 2007;14(3):1020-3.

20. Laronga C, Hasson D, Hoover S, Cox J, Cantor A, Cox C, et al. Paget's disease in the era of sentinel lymph node biopsy. Am J Surg. 2006;192(4):481-3

21. Zheng S, Song QK, Zhao L, Huang R, Sun L, Li J, et al. Characteristics of mammary Paget's disease in China: a nationalwide multicenter retrospective study during 1999-2008 Asian Pac J Cancer Prev. 2012;13(5):1887-93.

22. Lester T, Wang J, Bourne P, Yang Q, Fu L, Tang P. Different panels of markers should be used to predict mammary Paget's disease associated with in situ or invasive ductal carcinoma of the breast. Ann Clin Lab Sci. Winter 2009;39(1):17-24

23. Alikanoglu AS, Yildirim M, Suren D, Tutus B, Kaya V, Topal CS, et al. Expression of Cox-2 and Bcl-2 in Paget's disease of the breast Asian Pac J Cancer Prev. 2015;16(3):1041-5.

24. Ling $H, H u X, X u X L$, Liu ZB, Shao ZM. Patients with nippleareola Paget's disease and underlying invasive breast carcinoma have very poor survival: a matched cohort study. PLoS One. 2013;8(4):e61455.

25. Lohsiriwat V, Martella S, Rietjens M, Botteri E, Rotmensz N, Mastropasqua MG, et al. Paget's disease as a local recurrence after nipple-sparing mastectomy: clinical presentation, treatment, outcome, and risk factor analysis. Ann Surg Oncol. 2012;19(6): 1850-5. 